\title{
Pater Wilhelm Schmidt und Sigmund Freud: Gesellschaftliche Kontexte einer religionsethnologischen Kontroverse in der Zwischenkriegszeit
}

\author{
Peter Rohrbacher $\mathbb{D}$
}

Eingegangen: 15. Juni 2020 / Angenommen: 19. Juli 2020 / Online publiziert: 26. August 2020

(C) Der/die Autor(en) 2020

Zusammenfassung Diese Studie beschäftigt sich mit den konträren Thesen zur Religionsentstehung, die Pater Wilhelm Schmidt und Sigmund Freud 1912/1913 in Wien vorlegten. Eine Auseinandersetzung fand viele Jahre nicht statt, bis Schmidt Ende Oktober 1928 Freud mit einem öffentlichen Vortrag herausforderte, den Freud nicht erwiderte. Freud bezeichnete Schmidt allerdings in den 1930er-Jahren in privaten Briefen als den Hauptfeind der Psychoanalyse in Wien und vermutete, dass Schmidt aufgrund seiner guten Kontakte zum Papst ein Verbot der Psychoanalyse im Vatikan initiierte. Ziel der Untersuchung ist die Herausarbeitung der gesellschaftlichen Kontexte dieser seit Langem bekannten Freud-Narrative. Die ersten Abschnitte erörtern die Gründe, weshalb Schmidts Kritik darauf abzielte, auf Freud das Feindbild des Bolschewismus zu übertragen. Dabei wird folgende Hypothese verfolgt: Schmidts Angriff auf Freud steht in Zusammenhang mit der Gründungsinitiative einer psychoanalytischen Privatlehranstalt in Wien, die im Januar 1929 unter dem christlich-sozialen Bildungsminister Richard Schmitz abgelehnt wurde. Der letzte Teil untersucht Dokumente aus dem „Heiligen Offizium“, das 1933/34 ein Buchverbotsverfahren gegen Freuds Schüler Edoardo Weiss führte. Die Studie basiert auf Schmidts Veröffentlichungen in Bezug auf Freud, ausgewählter Literatur von und über Freud, Zeitungsberichten aus Österreich sowie Archivmaterial aus dem Vatikan.

Schlüsselwörter Geschichte der Anthropologie · Geschichte der Psychoanalyse • Totem und Tabu $\cdot$ Feindbild des Bolschewismus · Faschismus

P. Rohrbacher $(\bowtie)$

Institut für Sozialanthropologie, Österreichische Akademie der Wissenschaften, Hollandstraße 11-13, 1020 Wien, Österreich

E-Mail: Peter.Rohrbacher@oeaw.ac.at 


\title{
Father Wilhelm Schmidt and Sigmund Freud: social contexts of a controversy in the anthropology of religion during the interwar period
}

\begin{abstract}
This study deals with the contrasting theses on the origin of religion that Father Wilhelm Schmidt and Sigmund Freud presented in Vienna in 1912/1913. A discussion did not take place for many years until Schmidt challenged Freud with a public lecture at the end of October 1928, which Freud did not respond to. In private letters in the 1930s, however, Freud described Schmidt as the main enemy of psychoanalysis in Vienna and suspected that Schmidt, because of his good contacts with the Pope, had initiated a ban on psychoanalysis in the Vatican. The aim of the study is to work out the social contexts of these long-known narratives of Freud's. The first sections discuss the reasons why Schmidt's criticism was aimed at portraying Freud as the enemy of Bolshevism. Thus, the following hypothesis is pursued: Schmidt's attack against Freud correlates with the founding initiative of a private psychoanalytic institute in Vienna, which was rejected in January 1929 under the Christian-Socialist minister of education Richard Schmitz. The last part examines documents from the "Holy Office", which in 1933/1934 conducted a book prohibition trial against Freud's student Edoardo Weiss. The study is based on Schmidt's publications relating to Freud, selected literature by and about Freud, newspaper reports from Austria and archival material from the Vatican.
\end{abstract}

Keywords History of anthropology $\cdot$ History of psychoanalysis · Totem and taboo $\cdot$ Enemy of Bolshevism $\cdot$ Fascism

\section{Père Wilhelm Schmidt et Sigmund Freud: contextes sociaux d'une controverse dans l'anthropologie de la religion pendant l'entre-deux- guerres}

Résumé «Père Wilhelm Schmidt et Sigmund Freud: Contextes sociaux d'une controverse dans l'anthropologie de la religion pendant l'entre-deux-guerres »: Cette étude traite des thèses contraires sur l'origine de la religion, présentées par le père Wilhelm Schmidt et Sigmund Freud à Vienne en 1912/1913. Une discussion n'a pas eu lieu pendant de nombreuses années jusqu'à ce que Schmidt conteste Freud par une conférence publique à la fin du mois d'octobre 1928, que Freud n'a pas reprise. Dans des lettres privées des années 1930, Freud décrit cependant Schmidt comme le « principal ennemi » de la psychanalyse à Vienne et soupçonne que Schmidt, en raison de ses bons contacts avec le pape, a initié une interdiction de la psychanalyse au Vatican. L'objectif de l'étude est de déterminer les contextes sociaux de ces récits de Freud connus de longue date. Les premières sections abordent les raisons pour lesquelles la critique de Schmidt visait à attribuer à Freud l'image ennemie du bolchevisme. L'hypothèse suivante est poursuivie: L'attaque de Schmidt contre Freud est corrélée à l'initiative fondatrice d'un institut psychanalytique privé à Vienne, qui a été rejetée en janvier 1929 par le ministre chrétien-social de l'éducation Richard Schmitz. La dernière partie examine les documents du «Saint-Office » qui, en 1933/34, a mené un procès pour interdiction de livre contre l'étudiant de Freud, Edoardo Weiss. L'étude est basée sur les publications de Schmidt relatives à Freud, 
sur une sélection d'ouvrages écrits par et sur Freud, sur des articles de journaux autrichiens et sur des documents d'archives du Vatican.

Mots-clés Histoire de l'anthropologie · Histoire de la psychanalyse · Totem et tabou $\cdot$ Ennemie du bolchevisme $\cdot$ Fascisme

Zur Entstehung der Religion hatten Pater Wilhelm Schmidt (1868-1954) und Sigmund Freud (1856-1939) völlig konträre Vorstellungen. Schmidt war davon überzeugt, dass Gott sich in der Frühzeit dem Menschen direkt und unmittelbar offenbart habe. Daraus leitete er die Existenz eines ursprünglichen Monotheismus ab (Schmidt 1912, S. 573; Figl 2003, S. 547). Freud hingegen war der Auffassung, am Anfang der menschlichen Kulturentwicklung stünden totemistische Vorstellungen. Der Monotheismus sei lediglich eine später entstandene Religionsform und als infantile Vatersehnsucht anzusehen (S. Freud 1913, S. 137f.; Zettler 2020, S. 153). Diese konträren Thesen waren jeweils 1912/1913 in Wien entstanden und stützten sich auf ethnographische Daten, die weder von Schmidt noch von Freud erhoben wurden. Ein gegenseitiger Austausch fand viele Jahre nicht statt, bis Schmidt 1928 Freud mit einem öffentlichen Vortrag herausforderte, den Freud allerdings nicht erwiderte.

Erst posthum wurde bekannt, dass sich Freud mit Schmidts religionsethnologischem Lehrgebäude doch auseinandergesetzt hatte, allerdings nur privat. Die Veröffentlichungen eines Teils der Briefwechsel Freuds Ende der 1950er-Jahre dokumentieren, dass er in Schmidt den eigentlichen Hemmschuh sah, warum sich die Psychoanalyse in Wien nicht konsolidieren konnte (Jones 1957/III, S. 205ff., S. 223; E. L. Freud 1960, S. 103, S. 141). Die Freud-Forschung zog daraus den Schluss: Es war Schmidt, der mit seinen einflussreichen Kontakten zum autoritären Ständestaat und zum Vatikan die psychoanalytische Arbeit in Österreich und in Italien maßgeblich behinderte. Da sich bei Schmidt nicht nur ein katholischer, sondern für eine befristete Phase seiner Biographie auch ein rassischer Antisemitismus nachweisen lässt (Mischek 2008, S. 477; Pape 2009), festigte sich das von Freud stammende Bild (E. L. Freud 1960, S. 141), Schmidt sei der „Hauptfeind“ der Wiener Psychoanalyse gewesen (Robertson 1991). Freuds Briefvermerke bildeten schließlich auch die Grundlage für die Behauptung, Freud habe seine Romantrilogie Der Mann Moses als eine Gegenthese zu Schmidts Ursprung der Gottesidee im Britischen Exil vervollständigt (Robertson 1993, S. 349).

Diese Freud-Narrative wurden bisher nie aus der Perspektive Schmidts dargestellt. Teilaspekte dieser Lücken zu schließen, ist das Anliegen des vorliegenden Beitrags. Da vieles von der religionsethnologischen Kontroverse inhaltlich inzwischen überholt ist, wird der Fokus auf den gesellschaftspolitischen Kontext gelegt. Eine zentrale Bedeutung kommt der Fragestellung zu, ob Schmidt im Heiligen Offizium tatsächlich Maßnahmen gegen Freud initiierte. Für die vorliegende Studie wurden Schmidts Veröffentlichungen in Bezug auf Freud, ausgewählte Literatur von und über Freud, Zeitungsberichte aus Österreich sowie Archivalien aus dem Vatikan herangezogen. 


\section{Pater Wilhelm Schmidts Kritik an der Psychoanalyse Sigmund Freuds, 1928}

Schmidt hielt am 28. November 1928 in Wien den Vortrag „Die ethnologischen Grundlagen der Psychoanalyse - eine kritische Prüfung“. Die Veranstaltung, organisiert von der Kulturwissenschaftlichen Gesellschaft der Universität Wien, fand reges Interesse. Der Hörsaal für Histologie in der Schwarzspanierstraße 17 war schon vor Beginn der festgesetzten Stunde voll besetzt, das Medienecho danach gewaltig. Textauszüge des Vortrags wurden in mehreren Wiener Zeitungen kommentiert nachgedruckt (Schmidt 1929a; Anonym 1929b), vollständig allerdings nur im Jahrgang 1929 der Berliner Zeitschrift Nationalwirtschaft unter dem abgeänderten Titel „,Der Ödipus-Komplex der Freudschen Psychoanalyse und die Ehegestaltung des Bolschewismus“. Die Kontextualisierung des Vortrags erweist sich als nicht gerade einfach. Der Veranstalter verstand sich ausdrücklich als liberal und parteiunabhängig. Zu den Gründungsmitgliedern der von 1925 bis 1932 bestehenden Kulturwissenschaftlichen Gesellschaft zählten Moritz Schlick und Friedrich Hertz (Anonym 1925, S. 23). Die Herausgeber der Nationalwirtschaft hingegen, zu denen auch Othmar Spann zählte, bekämpften den Liberalismus und schenkten autoritären und faschistischen Strömungen ihre besondere Aufmerksamkeit. Die mediale Rezeption des Vortrags schließlich erfolgte ganz und gar über katholische Kanäle.

Schmidt stellte in seinem Vortrag die ethnologischen Grundlagen der Freud'schen Theorie des Ödipuskomplexes vor, wie Freud sie in seinem Buch Totem und Tabu (1913) dargelegt hatte. Seine Kritik baute auf der Arbeit des US-amerikanischen Anthropologen Alfred Kroeber auf, der aus ethnologischer Sicht 1920 bereits zehn Schwachstellen zu Freuds Kulturtheorie beanstandet hatte. Schmidts Prüfung berücksichtigte auch die Ergebnisse der kulturhistorischen Ethnologie, weshalb er Kroebers ,zehn knappen aber durchdringenden Thesen“ (Schmidt 1929b, S. 11) noch fünf weitere Sätze hinzufügte. Die Kritikpunkte betrafen hauptsächlich den sogenannten Totemismus, einen damals zentralen Begriff in der historisch orientierten Ethnologie, weil er mit den Anfängen der Religion in Verbindung gebracht wurde. Für Freud repräsentierte der Totemismus die Urreligion der Menschheit. Dabei stützte er sich auf ethnologische Gewährsmänner wie Edward B. Tylor und James G. Frazer, die einen universellen Evolutionismus vertraten. Für Kroeber und Schmidt hingegen war der Totemismus nichts Ursprüngliches, sondern bloß etwas Abgeleitetes und somit eine jüngere Religionsform. Auf die Pygmäen-Forschung in Zentralafrika und in Südostasien hinweisend erinnerte Schmidt daran, dass bei vielen Jäger- und Sammlergesellschaften der Totemismus nicht vorkomme und daher „keine allgemeine, alle Völker umfassende Durchgangsstufe“ sei (Schmidt 1929b, S. 12). Ende der 1920er-Jahre galt der ethnologische Evolutionismus allgemein als überholt, weshalb Freuds Kulturtheorie ein gewisser Anachronismus anhaftete. Dieser zeigte sich auch dahingehend, dass Freud seinen Aufsatz Totem und Tabu 1922 in dritter und 1925 in vierter Auflage in Wien abdrucken ließ, ohne dabei die in derselben Stadt begründete kulturhistorische Ethnologie mit einzubeziehen. Ein solches Vorgehen war ,in keiner Weise mehr wissenschaftlich gerechtfertigt“, konstatierte Schmidt (1929b, S. 7), was für ihn ein ausschlaggebender Grund war, überhaupt gegen Freud öffentlich Stellung zu beziehen. Diese sachlich geführte wis- 
senschaftliche Auseinandersetzung mit Freud nahm Schmidt (1930e, S. 106-111) schließlich in sein Handbuch der vergleichenden Religionsgeschichte auf, das in mehrere Sprachen übersetzt wurde. In all den anderen wissenschaftlichen Werken von Schmidt blieb Freud unerwähnt (Schmidt und Koppers 1924; Schmidt 1937). Selbst in Schmidts zwölfbändigem Hauptwerk Der Ursprung der Gottesidee wird Freud nur ein einziges Mal beiläufig genannt (Schmidt 1955, Bd. IV, S. 480).

In den katholisch ausgerichteten Zeitungen wurde Schmidts Vortrag hingegen mit der Schlagzeile „Eine wissenschaftliche Abrechnung mit Freud und seiner Lehre“ hochstilisiert und somit recht einseitig wiedergegeben (Walk 1928; Anonym 1929b). Diesen Vorbehalt gegenüber Freud teilte auch der Osservatore Romano, die offizielle Zeitung des Vatikans, die Schmidts Vortrag am 9. Januar 1929 als „Psychoanalyse und Ethnologie. Absurde Hypothese und historische Realität über die Ursprünge von Familie und Religion“ betitelte (Anonym 1929a). Von einer Widerlegung im epistemologischen Sinn konnte freilich nicht die Rede sein, da sich alle Aussagen von Schmidt und Freud auf die Frühzeit des Menschen bezogen und somit Hypothesen waren, die sich weder falsifizieren noch verifizieren ließen. Aus heutiger Sicht erscheint die damals religionsethnologisch geführte Diskussion über die historische Stellung des Totemismus eigentlich obsolet, da sich herausstellte, dass totemistische Phänomene auf Verwandtschaft beruhen und nur einen rudimentären religiösen Hintergrund haben (Duelke und Müller 2005, S. 378).

Schmidts Vortrag schloss allerdings auch eine politisch hochaufgeladene Komponente mit ein, die lohnenswert ist, untersucht zu werden. Das grundlegend Neue an Schmidts Kritik war der bemerkenswerte Versuch, den Ödipuskomplex als Theorem mit dem bolschewistischen Sexualstrafrecht zu verschränken. Freud und die Sowjets, so Schmidts Überlegungen, hatten das gemeinsame Ziel vor Augen, die bürgerliche Familie als Keimzelle des Staats zu zerstören. Schmidt deutete den Ödipuskomplex als ideologisches Werkzeug des Bolschewismus, der die Freud'sche Lehre im Zuge der Sexualstrafrechtsreform in Sowjetrussland seiner Ansicht nach bereits verwirklicht habe. Dementsprechend zeichnete Schmidt ein grauenvolles Bild über die dortigen gesellschaftlichen Verhältnisse. Ehepaare würden sich ihrer Kinder entledigen, indem sie ihr Neugeborenes am Eingang einer staatlichen Kinderanstalt auf eine Drehscheibe legen. Von dort würde es von einer Person angenommen, mit einer Nummer versehen, damit es von den leiblichen Eltern ,,spurlos abgelöst und in die große Schar der ,Staatskinder" eingereiht“" werde. Schmidt behauptete, dass ,bereits $80 \%$ aller Kinder aus Sowjetkreisen so ,behandelt"“ werden (Schmidt 1929b, S. 19). Seine Ausführungen beruhten nach eigenen Angaben auf einem Beitrag, den er der Arbeiter-Zeitung entnommen hatte.

Der Zeitungsbericht handelte über einen Vortrag, der am 13. November 1928 über das Thema „Sexualstrafrecht in Sowjetrußland“ in Wien abgehalten wurde. Der Vortragende hieß Nikolaj Pasche-Oserski, stand dem Juristischen Institut in Kiew vor und galt als Spezialist für die sowjetische Strafrechtsreform. Mit der Psychoanalyse hatte der "Sowjetprofessor“ (Schmidt 1929b, S. 18) somit gar nichts zu tun. Auch Pasche-Oserskis Ausführungen deckten nur sehr wenig von dem ab, was Schmidt behauptete daraus entnommen zu haben. So wurde das Sexualstrafrecht staatlicherseits gelockert, die Unauflöslichkeit der Ehe abgeschafft und ein Zusammenleben zwischen Mann und Frau auch außerehelich ermöglicht, was den Unterschied zwi- 
schen ehelichen und unehelichen Kindern aufhob. Die Reform betraf darüber hinaus Inzest und Homosexualität, die nun nicht mehr strafrechtlich verfolgt wurden. Auch Abtreibungen wurden erlaubt, allerdings nur bis zum dritten Schwangerschaftsmonat. Schmidt ging es somit weniger darum, die Situation im sowjetischen Russland zu beschreiben, sondern vielmehr das Feindbild des Bolschewismus zu schüren und zugleich auszuweiten, indem er es auch auf Freud übertrug. Den Grund dazu gab Schmidt auch an. Freud hatte in seinem jüngsten Buch Die Zukunft einer Illusion (1927) die Religion aus der Kultur herausgelöst und als Illusion bezeichnet. Daraus zog Schmidt den Schluss, zwischen Freud und den Sowjets bestehe ,eine nichtwegzudisputierende, entente cordiale "“ (Schmidt 1929b, S. 21). Freud erachtete zwar Religion als ,infantile Wiederkehr des Totemismus“, somit als eine kulturelle Krankheitsneurose, die es zu überwinden galt. Dennoch war er ein Gegner des Bolschewismus, was spätestens 1930 dezidiert nachweisbar ist (E. L. Freud 1960, S. 33, S. 37; S. Freud 1996, S. 92). Die Psychoanalyse wurde in Sowjetrussland als Ausdruck von bürgerlichem Individualismus angefeindet. Wenige Monate nach Schmidts Vortrag wurde sie unter Stalin verboten (Lobner und Levitin 1978, S. 16ff.; Zapperi 2016, S. 96). Die Frage stellt sich somit, warum Schmidt dieses Feindbild überhaupt mit Freud in Verbindung brachte. Im Folgenden möchte ich dafür eine Hypothese vorstellen, die in der Literatur bisher noch nicht berücksichtigt wurde (E. L. Freud 1960; Federn und Wittenberger 1992; S. Freud 1996, S. Freud und Eitingon 2004).

\section{Das Verbot der psychoanalytischen Privatlehranstalt, 1928/1929}

Im Jahr 1928 plante die Wiener Psychoanalytische Vereinigung (WPV) eine Privatlehranstalt für Psychoanalyse in Wien zu gründen und zwar nach jenen Vorbildern, wie sie in Berlin, Frankfurt, Paris und London bereits bestanden. Das Gesuch zur Bewilligung wurde allerdings unter dem christlich-sozialen Unterrichtsminister Richard Schmitz abgelehnt. Außerdem wurde eine nachträgliche Beschwerde seitens der WPV beim Verwaltungsgerichtshof am 29. Januar 1929 als unbegründet abgewiesen. Sektionsrat Otto Skrbensky als Vertreter des Unterrichtsministeriums hatte sich auf ein eingeholtes Gutachten des Psychologen Karl Bühler berufen, das ablehnend war (Anonym 1929d, S. 2). Der berühmte Entwicklungspsychologe stand der Psychoanalyse äußerst kritisch gegenüber und bezeichnete Freud in seinem Buch Die Krise der Psychologie (1927) abwertend als einen „Stoffdenker“ (Friedrich 2018). Das linksliberale Blatt Der Wiener Tag monierte hinsichtlich des Verbots in bissigem Ton, es sei für das „,klerikal angehauchte Unterrichtsministerium sozusagen ein ,Fressen“" gewesen, „moderne Spassetteln, als die die Psychoanalyse in den Augen eines klerikal Denkenden erscheinen muß, womöglich zu verhindern" (Anonym 1929e, S. 4).

Vor diesem Hintergrund kann Schmidts kritischer Freud-Vortrag auch als Intervention gegen eine offizielle Institutionalisierung der Psychoanalyse in Wien gedeutet werden. Eine solche zu verhindern, dazu hatte Schmidt regen Anlass gehabt. Nur wenige Monate zuvor wurde das Institut für Völkerkunde gegründet, das Schmidts katholisch ausgerichtete Ethnologie als Lehrgebäude fest an der Universität Wien 
verankerte (Gingrich 2005; Geisenhainer im Druck). Ein privates Freud-Institut wäre in dieser ethnologischen Konsolidierungsphase nur störend gewesen, vor allem in ideologischer Hinsicht. Der Lehrausschuss des WPV hatte nämlich vorgesehen, dass an der neuen Lehranstalt für Psychoanalyse neben Helene Deutsch auch Hermann Nunberg und Wilhelm Reich unterrichten würden (Anonym 1929e, S. 4). Nunberg war aktiver Sozialdemokrat und Reich Kommunist, der keinen Hehl daraus machte, die Psychoanalyse mit dem Marxismus zu verbinden. Nach den Erinnerungen des Psychoanalytikers Richard Sterba sei Reich in seiner Wiener Zeit bis 1930 überzeugt gewesen, dass durch das „,sowjetische Experiment“ von kollektiver Kindererziehung außerhalb der Familie der Ödipuskomplex und damit die Neurosen abgeschafft werden könnten (S. Freud 1996, S. 92). Für das Jahr 1928 sind einschlägige Vorträge von Reich in Wiener sozialistischen und kommunistischen Zirkeln nachweisbar, die in der Roten Fahne angekündigt waren (Anonym 1928a, S. 6). Im Dezember 1928 gründete Reich nach dem Vorbild des deutschen Sexualpädagogen Max Hodann (Anonym 1928d, S. 3) die Sozialistische Gesellschaft für Sexualberatung und Sexualforschung, die in Wien sechs unentgeltliche Sexualberatungsstellen für Arbeiter und Angestellte unterhielt (Anonym 1929c, S. 3). Freud selbst hielt das Verhalten Reichs allerdings für sehr schädlich, da er seiner Ansicht nach die Psychoanalyse für die ,bolsch.[ewistische] Propaganda“ missbrauchte (S. Freud 1996, S. 207). Das waren gesellschaftspolitische Entwicklungen, die den Vertretern des „Schwarzen Wien“ (Wassermann 2014) nach dem Brand des Wiener Justizpalasts am 15. Juli 1927 ein Dorn im Auge waren. Schmidts Überlegung, Freud werde für das „Sowjetexperiment" instrumentalisiert, war somit doch evidenzbasiert oder zumindest nicht völlig aus der Luft gegriffen. Eine wichtige Fragestellung wäre somit die Klärung, ob Schmidt aufgrund dieser linksorientierten Freudianer Einfluss auf die Ministerien nahm, um die Gründung der psychoanalytischen Privatlehranstalt Wien zu verhindern. Dazu bedarf es allerdings archivalischer Erhebungen, die bisher nicht einmal ansatzweise durchgeführt wurden und im Rahmen dieses Beitrags auch nicht geleistet werden können.

\section{Pater Wilhelm Schmidt und der Sowjetbolschewismus}

Wichtig erscheint mir der Zusammenhang, dass Schmidts Kritik am Sowjetbolschewismus völlig unabhängig von Freud entstand und nach seinem Vortrag auch wieder von ihm abgekoppelt weitergeführt wurde. Das lässt den Schluss zu, dass für Schmidt die Auseinandersetzung mit Freud bloß einen intellektuellen Nebenschauplatz einnahm. Schmidt ging es in erster Linie darum, die mögliche Zusammenarbeit des linken Flügels der Wiener Sozialdemokratie mit dem Sowjetkommunismus zu verhindern. Schmidts mahnende Stimme war diesbezüglich nicht neu. Erstmals warnte er bereits - angesichts der Arbeiter- und Soldatenräte in Deutschland und Ungarn (Herbeck 2009) - auf dem Katholikentag in Wien am 26. September 1920 vor einer sowjetischen Einflussnahme in Österreich. Schmidts Vortrag, der im Zeichen der bevorstehenden Nationalratswahl (17. Oktober 1920) stand, forderte dazu auf, ,Deutschösterreich und besonders das deutsche Wien von der schmachvollen Fremdherrschaft des jüdischen Bolschewikentums zu befreien“ (Schmidt 1920, 
S. 43). Schmidt war diesbezüglich kein Einzelfall. Das Feindbild vom ,jüdischen Bolschewiken“, der in Zentraleuropa „Terror“ verbreite, war damals sehr populär und wurde beispielsweise auch in Großbritannien von Winston Churchill (1920) propagiert. Realpolitisch war Schmidts antijüdische Hetzrede maßlos überzogen, da die Kommunisten in Österreich niemals politische Bedeutung erlangten. Das hielt ihn jedoch nicht davon ab, seinen Kampf gegen den sowjetischen Bolschewismus weiter fortzusetzen. Recht verlässliche Informationen aus der Sowjetunion erhielt Schmidt über seinen jüngeren Ordensbruder Eduard Gehrmann. Als sich 1922 auch der Vatikan an den vom Völkerbund veranlassten Hilfsmaßnahmen gegen die drohende Hungersnot in der Sowjetunion beteiligte, wurde Gehrmann zum Leiter des päpstlichen Hilfswerks in Moskau ernannt. Während seines Einsatzes verhandelte Gehrmann als Vertreter des Vatikans mit der sowjetischen Volkskommissarin Olga Kameneva, der Schwester Trotzkis (Kraus 1963) und wurde Augenzeuge der sowjetischen Verfolgungen gegenüber der orthodoxen und katholischen Kirche. Im Jahr 1924 konnte Schmidt einen wichtigen Erfolg seiner Agenda verbuchen: Ein von ihm mitverfasstes und redigiertes antikommunistisches Promemoria, das die vatikanische Diplomatie zur Sowjetunion nachhaltig veränderte, wurde Pius XI. persönlich ausgehändigt (Stehle 1993, S. 78f.).

Vor diesem Hintergrund ist es verständlich, weshalb für Schmidt der Vortrag des zuvor erwähnten „Sowjetprofessors“ Pasche-Oserski ein Affront war. Seine Einladung nach Wien war über den Kinderarzt und sozialdemokratischen Kommunalpolitiker Josef Karl Friedjung erfolgt (Anonym 1928b, S. 4), der seit 1909 Mitglied der WPN war. Weitere Vorträge von Pasche-Oserski fanden auf Einladung der österreichischen Gesellschaft zur Förderung der Beziehungen mit der UdSSR statt (Anonym 1928c, S. 3). Zudem leiteten Friedjung und Pasche-Oserski nationale Komitees in der Weltliga für Sexualreform, einer im Umfeld des Berliner Sexualforschers Magnus Hirschfeld im Juli 1928 gegründeten Organisation, die eine umfassende Gesellschaftsreform auf der Basis einer aufgeklärten, naturwissenschaftlich fundierten Sexualmoral anstrebte. Durch Friedjungs Engagement wurde im September 1930 der dritte Weltkongress in Wien (nach Kopenhagen und London) veranstaltet (McEwen 2012, S. 180).

Es lässt sich feststellen, dass Schmidts Stellungnahmen gegen den Bolschewismus nach seinem Freud-Vortrag verstärkt zunehmen. Der Kontext dieser Öffentlichkeitsarbeit ist jedoch ein anderer. Anfang Februar 1930 richtete der Papst ein Schreiben an seinen Generalvikar Basilio Pompili, worin er auf die Gräuel der Christenverfolgung in Sowjetrussland hinwies. Zahlreiche Vertreter des katholischen und des anglikanischen Klerus folgten diesem Aufruf und nahmen weltweit Stellung zum bolschewistischen Vernichtungsfeldzug gegen alles Religiöse. In ihren Predigten wurde der Bolschewismus vielfach mit Satansherrschaft gleichgesetzt. Schmidts Ende Februar 1930 verfasster Aufsatz für die Reichspost Der Weltprotest gegen die Religionsverfolgung Sowjetrußlands (Schmidt 1930a) zeigt, dass sich auch er an dieser vatikanischen Medienkampagne beteiligte. In seiner von März bis Juni 1930 erstellten dreiteiligen Artikelserie in der Schöneren Zukunft versuchte er die Gründe des bolschewistischen Religionshasses aufzudecken (Schmidt 1930b, c, d). Schmidt nahm dabei den bolschewistischen „Berufsrevolutionär“ ins Visier, der seine „Teufel“" in geheimer Mission nach Europa sende (Schmidt 1930b, S. 587, 1930c, S. 809). 
Freud und den Ödipuskomplex brachte Schmidt aber nunmehr nicht mehr in diesen Zusammenhang. Es bleibt festzuhalten, dass Schmidts ehemals laute Stellungnahmen gegen Freud nach 1928/1929 völlig verstummten.

\section{Pater Wilhelm Schmidt und Sigmund Freuds Denunziation im „Heilige Offizium“‘, 1933/34}

Erstaunlicherweise nahm Freud zu Schmidts Kritik nie öffentlich Stellung. Allerdings publizierte er im Jahr 1934 Totem und Tabu in Wien unverändert in fünfter Auflage, was auch als Protest gegen katholische Kritiker der Psychoanalyse wie Schmidt, Alois Mager (Ordo Sancti Benedicti; 1929) oder Oskar Herget $(1928,1934)$ gedeutet werden kann. Immerhin korreliert die Neuauflage zeitlich mit Freuds Briefeinträgen zu Schmidt, die sich für dieses Jahr erstmals nachweisen lassen (E. L. Freud 1960, S. 102f.). Freud hatte mit der Abfassung seiner Romantrilogie Der Mann Moses und die monotheistische Religion nach Hitlers Machtergreifung in Deutschland und dem damit zusammenhängenden Judenhass begonnen (E. L. Freud 1960, S. 102; Yerushalmi 1993, S. 39). Darin stellte Freud die historische Hypothese auf, dass Moses kein Jude, sondern ein Ägypter hoher Abkunft zur Zeit des Ketzerkönigs Echnathon gewesen sei und demzufolge nicht die Juden, sondern die alten Ägypter als „Erfinder" des Monotheismus anzusehen seien (Yerushalmi 1993; Assmann 1998). Das Manuskript war im September 1934 fertiggestellt (S. Freud 1996, S. 309). Freud (1937a, b) publizierte die ersten beide Teile, hielt jedoch den dritten Teil vor einer Veröffentlichung zurück, da er befürchtete, Schmidt würde in Österreich durch seine guten Kontakte zur austrofaschistischen Regierung (Mayer 1991, S. 123) die psychoanalytische Arbeit behindern. „Denn wir leben hier in einer Atmosphäre katholischer Strenggläubigkeit“, schrieb Freud besorgt bezugnehmend auf seinen neuen Roman, am 29. September 1934 an Arnold Zweig und führte weiter aus: „Man sagt, daß die Politik unseres Landes von einem Pater Schmidt gemacht wird, der in St. Gabriel bei Mödling lebt, der Vertrauensmann des Papstes ist und zum Unglück selbst ein Ethnolog und Religionsforscher, der in seinen Büchern aus seinem Abscheu vor der Analyse und besonders meiner Totemtheorie kein Geheimnis macht" (E. L. Freud 1960, S. 103). Freud vollendete sein letztes Originalwerk erst im Sommer 1939 im Londoner Exil. Die Romantrilogie erschien in Amsterdam, in einem Verlag, der sich auf deutschsprachige Exilliteratur spezialisiert hatte (S. Freud 1939).

Tatsächlich befand sich die Psychoanalyse in Österreich während der DollfußSchuschnigg-Periode (1933-38) in einer schwierigen Situation. Einerseits war ein staatliches Verbot durch die beherrschende katholische Übermacht absehbar. Andererseits schützte genau dieser politische Katholizismus die Psychoanalyse vor dem Nationalsozialismus (Huber 1977, S. 88). Freud sah allerdings nicht im Nationalsozialismus, sondern in der römisch-katholischen Kirche den Hauptgegner der Psychoanalyse. Als René Laforgue bei einem Besuch 1937, nur wenige Monate vor dem „Anschluss“ Österreichs an NS-Deutschland, Freud dazu riet das Land zu verlassen, reagierte Freud fast mit Verachtung: „Die Nazis? Ich habe keine Angst vor ihnen. Helfen Sie mir lieber, meinen wahren Feind zu bekämpfen." Als der Begründer der Psychoanalyse in Frankreich erstaunt nachfragte, um welchen Feind es sich hand- 
le, antwortete Freud: „Religion, die römisch-katholische Kirche“ (Laforgue 1973 [1956], S. 343f.).

Freuds Sorge bezog sich auf den Vatikan. Im Frühjahr 1934 hatte er von seinem Schüler Edoardo Weiss die Nachricht aus Rom erhalten, ,die kirchlichen Autoritäten [planten] die Einstellung unserer Zeitschrift" (S. Freud und Weiss 1973, S. 89). Es handelte sich hierbei um die psychoanalytische Fachzeitschrift Rivista italiana die psicoanalisi, die 1932 von Weiss in Rom mitbegründet wurde. Die Veröffentlichung war allerdings nur von kurzer Dauer, da die Zeitschrift bereits nach zwei Jahren von den faschistischen Behörden unter dem Druck der kirchlichen Hierarchien geschlossen wurde.

Das Verbot war ein schwerer Schlag gegen die Bewegung, immerhin war Weiss der „Pionier der Psychoanalyse in Italien“ (S. Freud und Weiss 1973, S. 38). Weiss, 1889 in Triest geboren, lernte Freud während seines Medizinstudiums in Wien kennen und wurde von Paul Federn analysiert. Im Jahr 1919 kehrte er in seine Heimatstadt zurück und erhielt eine Stelle in der dortigen psychiatrischen Provinzklinik. Während dieser Zeit übertrug er viele Werke Freuds ins Italienische, darunter auch Totem und Tabu (1930). Im Jahr 1931 wurde Weiss allerdings von den Faschisten aus Triest vertrieben und der italienische Psychiater Camillo Sante de Sanctis, mit dem er Korrespondenz führte, rief ihn nach Rom (Desmazières 2009, S. 87).

Freud schrieb im September 1934 an Arnold Zweig, dass das Verbot der Fachzeitschrift „direkt vom Vatikan“ ausgegangen und „Pater Schmidt“ dafür verantwortlich zu machen sei (E. L. Freud 1960, S. 103). Wenn also Schmidt ein solches Verbot über den Vatikan erwirkt haben soll, müssten sich in erster Linie Spuren im Heiligen Offizium, der obersten Glaubensbehörde des Vatikans finden lassen. Entsprach ein Buch nicht der katholischen Lehre, wurde es dort durch ein Verfahren auf den Index der verbotenen Bücher gesetzt. Die Anzeige konnte entweder aus der Kurie selbst oder von außerhalb kommen. Da das Archiv der Kongregation für die Glaubenslehre der historischen Forschung erst 1998 zugänglich gemacht wurde, blieben Freuds Mutmaßungen trotz kritischer Anmerkungen (Yerushalmi 1993, S. 165) lange Zeit ungeprüft.

Pater Wilhelm Schmidt (Societas Verbi Divini) war als Direktor des Päpstlichen Museo missionario-etnologico bestens im Vatikan vernetzt, galt als Vertrauter des Papstes und genoss zudem auch hohes Ansehen an der Kurie. Im Jahr 1933 wurde Schmidts Handbuch der vergleichenden Religionsgeschichte, das die ethnologische Kritik von Freuds Totemtheorie enthielt, vom katholischen Verlagshaus Morcelliana in Brescia ins Italienische übersetzt. Darüber hinaus ist inzwischen auch bekannt, dass Schmidt 1936 als Konsulent für das Heilige Offizium herangezogen wurde, um Fragen über den Evolutionismus aus ethnologischer Sicht für die Kurienmitglieder abzuklären (Rohrbacher 2012). Diese Auflistung verdichtet somit Freuds Mutmaßung, wonach Schmidt eine treibende Kraft gegen die Psychoanalyse im Vatikan gewesen sein könnte. Tatsächlich ließ sich im Archiv der Kongregation für die Glaubenslehre ein Bestand identifizieren, der über die Beschäftigung des Heiligen Offiziums mit Freuds Psychoanalyse von Februar 1933 bis April 1934 minutiös Auskunft gibt. Der Bestand wurde von Agnés Desmazières, einer französischen Religionshistorikerin der jesuitischen Privatuniversität Centre Sèvres in Paris, erstmals ausgewertet. Da ihr Urteil in Bezug auf Schmidts Rolle im Heiligen Offizium 
indifferent ausfällt (Desmazières 2009, S. 89f.), hielt ich es für nötig, dieses vatikanische Archiv 2018 selbst zu konsultieren. Das wichtigste Ergebnis der erneuten Einsichtnahme sei vorweggenommen: Der Name Wilhelm Schmidt kommt im gesamten Bestand nicht vor. Die inhaltliche Auseinandersetzung mit der Freud'schen Psychoanalyse oblag den Jesuiten.

Die Neuauflage des erfolgreichen Buchs Elementi di Psicoanalisi (1931) von Edoardo Weiss veranlasste das Heilige Offizium, sich mit der Psychoanalyse zu befassen. Das Werk, das auf einer Vortragssammlung der Associazione Medica Triestina basierte und ein empfehlendes Geleitwort von Freud enthielt, popularisierte die Psychoanalyse in Italien. Das Buch wurde von Monsignore Giuseppe Latini denunziert, der im Heiligen Offizium die Rolle eines „Förderers der Gerechtigkeit““ einnahm. Nach eigenen Angaben hatte Latini es im „Fenster eines Buchhändlers“ entdeckt. ${ }^{1}$ Im Februar 1933 bat die Heilige Kongregation Francesco Gaetani (Societas Jesu), ein Votum nicht nur über die Elementi di psicoanalisi, sondern auch über die Psychoanalyse im Allgemeinen zu erstellen. Der jesuitische Konsulent ließ sein Ergebnis zuerst von seinem Ordensgeneral prüfen, der in diesem Buchverbotsverfahren eine Schlüsselrolle einnahm.

Wladimir Ledóchowski, der jesuitische General mit österreichisch-polnischen Wurzeln, teilte Gaetanis Kritik der Psychoanalyse mit seiner Empfehlung zu ihrer Verurteilung. Er hielt es aber für notwendig, Gaetanis Text ,im Geheimen“, wie er betonte, zusätzlich auch von Josef Donat (Societas Jesu), Professor für christliche Philosophie an der Jesuitenuniversität Innsbruck, revidieren zu lassen. ${ }^{2}$ Der österreichische Jesuit, der im Jahr zuvor eine Widerlegung der Psychoanalyse veröffentlicht hatte (Donat 1932), billigte das verurteilende Votum Gaetanis, indem es hieß: „Anstatt die Verurteilung auf das Weiss-Buch im Besonderen zu beschränken, erscheint es mir daher angemessener, sie auf die Freudsche Psychoanalyse im Allgemeinen auszudehnen“"3 Als Ledóchowski die beiden jesuitischen Stellungnahmen dem Heiligen Offizium übergab, vermerkte er am 22. Mai 1933 an den Assessor Nicola Canali: „(...) [Es] wäre sehr wünschenswert, dass die oberste Autorität der Kirche in dieser Angelegenheit eine Entscheidung trifft. Offensichtlich könne kein Katholik, geschweige denn ein Priester die materialistischen Theorien Freuds akzeptieren“. Aus diesem Grund müsse er, betonte Ledóchowski im selben Schreiben an den höchsten Beamten der vatikanischen Glaubensbehörde, ,einige unserer Patres vor dieser Gefahr warnen“. ${ }^{4}$ Das ist ein deutliches Indiz dafür, dass nicht Schmidt das Verbot der Freud'schen Psychoanalyse im Heiligen Offizium vorantrieb, sondern der Jesuitengeneral.

Der weitere Ablauf des Verfahrens verzögerte sich indes empfindlich. Die nächste Sitzung am Heiligen Offizium wurde erst Monate später am 9. April 1934 einberufen und ergab kein endgültiges Ergebnis. Die Teilnehmer, darunter auch der österrei-

\footnotetext{
1 Archivio della Congregazione della Fede (ACDF), Censurae Librorum Sanctum Officium (Città del Vaticano), CL, 248 (1933), fol. 39; Latini, 08.04.1934. Alle aus diesem Archiv stammenden Zitate wurden vom Verfasser aus dem Italienischen übersetzt.

2 ACDF, CL, 248 (1933), fol. 3; Ledóchowski, 01.05.1933, an Canali.

3 ACDF, CL, 248 (1933), fol. 5; Gaetini, gedrucktes Votum vom Juni 1933, S. 1-20, hier S. 15.

4 ACDF, CL, 248 (1933), fol. 4; Ledóchowski, 22.05.1933, an Canali.
} 
chische Bischof Alois Hudal, einigten sich darauf, die zusätzliche Meinung eines medizinischen Experten heranzuziehen. Zuerst müsse Gewissheit erlangt werden, lautete das zusammenfassende Urteil, was ,,an der Freudschen Theorie möglicherweise oder tatsächlich wahr ist" ${ }^{5}$ Eine letzte Sitzung wurde für den 18. April 1934 angesetzt, sie kam allerdings aus unbekannten Gründen nicht zustande. Trotz der ablehnenden Voten seitens der Jesuiten verurteilte das Heilige Offizium weder das Buch von Weiss noch die Freud'sche Psychoanalyse. Vermutlich wurde das Verfahren eingestellt, weil die faschistischen Behörden bereits „Ende 1933“ (S. Freud und Weiss 1973, S. 35), wie Weiss in seinen Erinnerungen an Freud festhielt, ein Verbot erließen, die Fachzeitschrift Rivista italiana die psicoanalisi weiter zu veröffentlichen. Obwohl Weiss einen ,guten Zugang zu Mussolini“" hatte, wie Freud im September 1934 gegenüber Zweig attestierte (E. L. Freud 1960, S. 103; Mehler 2010, S. 133), stand Weiss nach eigenen Angaben stets in „Opposition zum Faschismus“ (S. Freud und Weiss 1973, S. 35). Schmidt war somit an diesem vatikanischen Buchverbotsverfahren gänzlich unbeteiligt, ein Befund, der sich auch mit den Aufzeichnungen von Weiss deckt: Weder in seinen Briefen noch in seiner Erinnerung wird der Name Wilhelm Schmidt genannt (S. Freud und Weiss 1973). Durch diese klärende Feststellung wird nicht ausgeschlossen, dass sich Schmidt im Vatikan auch über andere Kanäle gegen Freud oder Weiss eingesetzt haben könnte. Dazu bedarf es allerdings weiterer Forschungsarbeit in den anderen vatikanischen Archiven. Eine vielversprechende Spur führt zu Pater Pietro Tacchi Venturi (Societas Jesu) (Turi 2002, S. 92), dem geheimen Mittelsmann zu Mussolini. Diesbezügliche Nachforschungen blieben bisher jedoch ergebnislos (Zapperi 2016, S. 101; Kertzer 2016).

\section{Conclusio}

Als Ergebnis dieser Gegenüberstellung kann festgehalten werden, dass die eingangs genannten Freud-Narrative in einem neuen und differenzierten Licht zu sehen sind. Freuds sorgenvolles Bild, Schmidt sei der Hauptfeind der Wiener Psychoanalyse in den Jahren 1934 und 1936 gewesen, findet bei Schmidt keine Entsprechung. Nach 1930 sind bei Schmidt keine öffentlichen Stellungnahmen gegen Freud in Form von Vorträgen oder Publikationen feststellbar. Wie gezeigt werden konnte, nimmt Freuds Kulturtheorie, wie sie in Totem und Tabu dargelegt ist, in Schmidts wissenschaftlichem Werk einen nur sehr marginalen Platz ein.

Nachdem Kroeber (1920) seine harsche Kritik im American Anthropologist vorgelegt hatte, galt Freuds Totem und Tabu seitens der Ethnologie größtenteils als obsolet. Warum sich Schmidt dennoch mit diesem Werk auseinandersetzte, hing mit dem gesellschaftspolitischen Kontext des Jahres 1928 zusammen. In diesem Jahr wurde das Institut für Völkerkunde an der Universität Wien gegründet. Ebenso war zu dieser Zeit in Wien die Gründung eines privaten „Freud-Instituts“ vorgesehen, dessen Lehrpersonal sich allerdings aus vorwiegend linksorientierten Freudianern zusammensetzte. Es konnten mehrere Indizien gefunden werden, dass Schmidts Vortrag,

\footnotetext{
5 ACDF, CL, 248 (1933), fol. 38; Feria II, 09.04.1934.
} 
der beabsichtigte, das Feindbild des Bolschewismus auf Freud zu übertragen, sich auf diese Gründungsinitiative bezog. Die Fragestellung, ob Schmidt auch auf Ministerialebene intervenierte, blieb offen, ebenso die Klärung der Hintergründe, weshalb es 1928/1929 überhaupt zu einem Verbot der psychoanalytischen Privatlehranstalt in Wien kam.

Das wohl wichtigste Teilergebnis dieses Beitrags bezieht sich auf den Vatikan. Meine auf Desmazières' aufbauenden vatikanischen Nachforschungen ergaben: Schmidt hat weder Sigmund Freud noch seinen Schüler Edoardo Weiss im Heiligen Offizium angezeigt. Das Buchverbotsverfahren zu Edoardo Weiss in der Glaubenskongregation trieben Jesuiten (letztlich vergebens) voran. Diese klärende Feststellung schließt allerdings nicht aus, dass sich Schmidt im Vatikan auch über andere Kanäle gegen die Psychoanalyse eingesetzt haben könnte.

Danksagung Für Anregungen zur Verbesserung dieses Beitrags danke ich Andre Gingrich (ÖAW).

Förderung Diese Studie wurde aus Mitteln des Austrian Science Fund (FWF) mit der Projektnummer P 33427-G gefördert.

Open Access Dieser Artikel wird unter der Creative Commons Namensnennung 4.0 International Lizenz veröffentlicht, welche die Nutzung, Vervielfältigung, Bearbeitung, Verbreitung und Wiedergabe in jeglichem Medium und Format erlaubt, sofern Sie den/die ursprünglichen Autor(en) und die Quelle ordnungsgemäß nennen, einen Link zur Creative Commons Lizenz beifügen und angeben, ob Änderungen vorgenommen wurden.

Die in diesem Artikel enthaltenen Bilder und sonstiges Drittmaterial unterliegen ebenfalls der genannten Creative Commons Lizenz, sofern sich aus der Abbildungslegende nichts anderes ergibt. Sofern das betreffende Material nicht unter der genannten Creative Commons Lizenz steht und die betreffende Handlung nicht nach gesetzlichen Vorschriften erlaubt ist, ist für die oben aufgeführten Weiterverwendungen des Materials die Einwilligung des jeweiligen Rechteinhabers einzuholen.

Weitere Details zur Lizenz entnehmen Sie bitte der Lizenzinformation auf http://creativecommons.org/ licenses/by/4.0/deed.de.

\section{Literatur}

Anonym. 1925. Eine neue akademische Gesellschaft. Neues Wiener Journal. Unparteiisches Tagblatt 33:23. 15.2.1925.

Anonym. 1928a. Freie Vereinigung sozialistischer Studenten. Die rote Fahne. Zentralorgan der kommunistischen Partei Österreichs 11(59):6. 9.3.1928.

Anonym [Dr. P.St.]. 1928b. Das Sexualstrafrecht in Sowjetrussland. Arbeiter-Zeitung. Zentralorgan der Sozialdemokratie Deutschösterreichs 41:4. 16.11.1928.

Anonym. 1928c. Weitere Vorträge des Genossen Prof. Oserski. Die Rote Fahne. Zentralorgan der Kommunistischen Partei Oesterreichs 11:3. 18.11.1928.

Anonym. 1928d. Sexualelend, Sexualberatung, Sexualrevolution. Genosse Dr. Max Hodann in Wien. Die Rote Fahne. Zentralorgan der Kommunistischen Partei Oesterreichs 11:3. 19.12.1928.

Anonym [G. B.]. 1929a. Psicoanalisi ed Etnologia. Ipotesi assurde e realità storica sulle origini della famiglia e della religione. L'Osservatore Romano (Città del Vaticano) 69(7):2-3. 9.1.1929.

Anonym. 1929b. Univ.-Prof. Dr. P. Wilh. Schmidt S.V.D.: Eine wissenschaftliche Abrechnung mit der Psychoanalyse. Das Neue Reich. Wochenschrift für Kultur Politik und Volkswirtschaft 11:266-267. 12.1.1929.

Anonym. 1929c. Sexualberatungsstellen. Arbeiter-Zeitung. Zentralorgan der Sozialdemokratie Deutschösterreichs 42:3. 21.1.1929.

Anonym. 1929d. Die verbotene Privatlehranstalt für Psychoanalyse. Die Stunde 7:2. 31.1.1929. 
Anonym. 1929e. Psychoanalyse - eine noch nicht anerkannte Wissenschaft. Warum die Privatlehranstalt für Psychoanalyse nicht gegründet werden kann. Der Wiener Tag 8:4. 31.1.1929.

Archivio della Congregazione della Fede (ACDF). 1933. Censurae Librorum Sanctum Officium (Città del Vaticano), CL, 248

Assmann, J. 1998. Moses der Ägypter. Entzifferung einer Gedächtnisspur. München: Hanser.

Bühler, K. 1927. Die Krise der Psychologie. Jena: Gustav Fischer.

Churchill, W. 1920. Zionism versus Bolshevism. A Struggle for the Soul of the Jewish People. Illustrated Sunday Herald 8:5.

Desmazières, A. 2009. La psychanalyse àl'Index? Sigmund Freud aux prises avec le Vatican (1921-1934). Vingtième Siècle. Revue d'histoire 102:79-91.

Donat, J. 1932. Über Psychoanalyse und Individualpsychologie. Innsbruck: Rauch.

Duelke, B., und W. Müller. 2005. Totemismus. In Wörterbuch der Völkerkunde, 2. Aufl., Hrsg. W. Hirschberg, W. Müller, 377-378. Berlin:: Reimer. Neuausgabe.

Federn, E., und G. Wittenberger (Hrsg.). 1992. Aus dem Kreis um Sigmund Freud. Zu den Protokollen der Wiener Psychologischen Vereinigung. Frankfurt/Main: Fischer.

Figl, J. 2003. Gott - monotheistisch. In Handbuch Religionswissenschaft. Religionen und ihre zentralen Themen, Hrsg. J. Figl, 545-558. Innsbruck: Tyrolia Verlagsanstalt.

Freud, E.L. (Hrsg.). 1960. Sigmund Freud/Arnold Zweig, Briefwechsel. Frankfurt/Main: Fischer.

Freud, S. 1913. Totem und Tabu: einige Übereinstimmungen im Seelenleben der Wilden und der Neurotiker. Leipzig, Wien: Heller. orig. 1912-13.

Freud, S. 1927. Die Zukunft einer Illusion. Leipzig, Wien, Zürich: Internationaler Psychoanalytischer Verlag.

Freud, S. 1937a. Moses ein Ägypter. Imago. Zeitschrift für Anwendung der Psychoanalyse auf die Geisteswissenschaften XXIII:5-13.

Freud, S. 1937b. Wenn Moses ein Ägypter war. Imago. Zeitschrift für Anwendung der Psychoanalyse auf die Geisteswissenschaften XXIII:387-419.

Freud, S. 1939. Der Mann Moses und die monotheistische Religion. Drei Abhandlungen. Amsterdam: de Lange.

Freud, S. 1996. Tagebuch 1929-1939: kürzeste Chronik. Basel, Frankfurt/Main: Stroemfeld. hrsg. und eingeleitet von M. Molnar.

Freud, S., und M. Eitingon. 2004. Briefwechsel 1906-1939. Bd. 2. Tübingen: edition diskord. hrsg. von M. Schröter.

Freud, S., und E. Weiss. 1973. Briefe zur psychoanalytischen Praxis. Mit den Erinnerungen eines Pioniers der Psychoanalyse. Reihe Fischer, Bd. 34. Frankfurt/Main: Fischer. Vorbemerkung und Einleitung von M. Grotjahn.

Friedrich, J. (Hrsg.). 2018. Karl Bühlers Krise der Psychologie. Positionen, Bezüge und Kontroversen im Wien der 1920er/30er Jahre. Veröffentlichungen des Instituts Wiener Kreis, Bd. 26. Cham: Springer.

Geisenhainer, K. in Druck. Gescheiterte Interventionen: Otto Reche und seine Wiener Nachfolge 1926-1928 (Exkurs). In Völkerkunde zur NS-Zeit aus Wien (1938-1945): Institutionen, Praktiken und Biographie-zentrierte Netzwerke, Hrsg. A. Gingrich, P. Rohrbacher. Wien: ÖAW.

Gingrich, A. 2005. The German-speaking countries. Ruptures, schools, and nontraditions: reassessing the history of sociocultural anthropology in Germany. In One discipline, four ways: British, German, French, and American anthropology, Hrsg. F. Barth, A. Gingrich, R. Parkin, und S. Silverman, 61-153. Chicago, London: University of Chicago Press.

Herbeck, U. 2009. Das Feindbild vom „jüdischen Bolschewiken“. Zur Geschichte des russischen Antisemitismus vor und während der Russischen Revolution. Berlin: Metropol.

Herget, O. 1928. Eine Abrechnung mit Freud, Zu Sigm. Freuds jüngstem Werke: „Die Zukunft einer Illusion“. Reichspost. Unabhängiges Tagblatt für das christliche Volk 118(35):7. 28. April 1928.

Herget, O. 1934. Buchbesprechung zum „Almanach der Psychoanalyse 1934“. Reichspost. Unabhängiges Tagblatt für das christliche Volk 41(110):7. 23.4.1934.

Huber, W. 1977. Psychoanalyse in Österreich seit 1933. Bd. 2. Wien, Salzburg: Geyer.

Jones, J. 1957. Sigmund freud. Life and work. London: The Hogarth Press. Three Volumes.

Kertzer, D.I. 2016. Der erste Stellvertreter. Pius XI. und der geheime Pakt mit dem Faschismus. Darmstadt: Theiss. orig. 2014.

Kraus, J. 1963. Der Sekretär zweier Nuntien. Pater Eduard Gehrmann (Societas Verbi Divini). In Verbo Tuo. Festschrift zum 50jährigen Bestehen des Missionspriesterseminars St. Augustin bei Siegburg/ Rheinland 1913-1963 Veröffentlichungen des Missionspriesterseminars St. Augustin, Bd. 12, Hrsg. Lektoren in St. Augustin, 167-195. Steyl und Kaldenkirchen: Steyler Verlag.

Kroeber, A.L. 1920. Totem and taboo. An ethnologic psychoanalysis. American Anthropologist 22:48-55. 
Laforgue, R. 1973. Personal memories of Freud (1956). In Freud as we knew him, Hrsg. H.M. Ruitenbeek, 341-349. Detroit: Wayne State University Press.

Lobner, H., und V. Levitin. 1978. A short account of Freudism. Sigmund Freud House Bulletin 2:5-29.

Mager, A. 1929. Psychoneurosen. Schönere Zukunft. Wochenschrift für Kultur und Politik, Volkswirtschaft und Soziale Frage IV(39):826-827. 30.6.1929.

Mayer, A. 1991. Die Völkerkunde an der Universität Wien bis 1938. Diplomarbeit, Universität Wien.

McEwen, B. 2012. Sexual knowledge: Feeling, fact, and social reform in Vienna, 1900-1934. Austrian and Habsburg studies, Bd. 13. New York: Berghahn Books.

Mehler, J.A. 2010. Psychoanalyse und Faschismus in Italien. In Psychoanalyse in totalitären und autoritären Regimen, Hrsg. M.G. Ash, 129-151. Frankfurt/Main: Brandes \& Apsel.

Mischek, U. 2008. Antisemitismus und Antijudaismus in den Werken und Arbeiten Pater Wilhelm Schmidts S.V.D. (1868-1954). In The study of religion under the impact of fascism, Hrsg. H. Junginger, 467-488. Leiden: Brill.

Pape, Ch . 2009. Wilhelm Schmidt. In Handbuch des Antisemitismus. Judenfeindschaft in Geschichte und Gegenwart Personen, Bd. 2, Hrsg. W. Benz, 738-739. Berlin: De Gruyter Saur.

Robertson, R. 1991. My true Enemy: Freud and the Church 1927-1939. In Austria in the thirties. Culture and politics Studies in Austrian literature, culture and thought., Hrsg. K. Segar, J.W. Riverside, 328-344. Riverside: Ariadne.

Robertson, R. 1993. Freud und Pater Wilhelm Schmidt. In Die Wiener Jahrhundertwende, Hrsg. J.P. Nautz, R. Vahrenkamp, 360-376. Wien, Köln, Graz: Böhlau.

Rohrbacher, P. 2012. Völkerkunde und Afrikanistik für den Papst. Missionsexperten und der Vatikan 1922-1939 Römische Historische Mitteilungen 54:583-610.

Schmidt, W. 1912. Die Uroffenbarung als Anfang der Offenbarungen Gottes. In Religion, Christentum, Kirche. Eine Apologetik für wissenschaftlich Gebildete, Bd. 1, Hrsg. G. Esser, J. Mausbach, 481-636. Kempten, München: Kösel'sche Buchhandlung.

Schmidt, W. 1920. Befreiung Wiens von jüdischem Bolschewismus! Eine Katholikentagsrede von Professor Dr. Wilhelm Schmidt S.V.D. Das Neue Reich. Wochenschrift für Kultur Politik und Volkswirtschaft 3:42-43. 10.10.1920.

Schmidt, W. 1929a. Prof. Dr. Freuds psychoanalytische Theorie zum Ursprung der Familie und der Religion. Eine kritische Prüfung ihrer ethnologischen Grundlagen. Schönere Zukunft. Wochenschrift für Kultur und Politik, Volkswirtschaft und Soziale Frage IV(13):263-265. Nr. 14 (6.1.1929), 287-289, Nr. 15 (13.1.1929), 308-310.

Schmidt, W. 1929b. Der Ödipus-Komplex der Freudschen Psychoanalyse und die Ehegestaltung des Bolschewismus. Eine kritische Prüfung ihrer ethnologischen Grundlagen. „Nationalwirtschaft“. Blätter für organischen Wirtschaftsaufbau. Berlin: Verlag der „Nationalwirtschaft“.

Schmidt, W. 1930a. Der Weltprotest gegen die Religionsverfolgung Sowjetrußlands. Reichspost. Unabhängiges Tagblatt für das christliche Volk 37(53):1. 23.2.1930.

Schmidt, W. 1930b. Die tieferen Untergründe des bolschewistischen Religionshasses. Schönere Zukunft. Wochenschrift für Kultur und Politik, Volkswirtschaft und Soziale Frage V(24):587-588. V, Nr. 34 (25.5.1930), 809-810.

Schmidt, W. 1930c. Die Gewissenlosigkeit der sowjetrussischen Experimente. Schönere Zukunft. Wochenschrift für Kultur und Politik, Volkswirtschaft und Soziale Frage V(35):835-836. 1.6.1930.

Schmidt, W. 1930d. Sowjetrußland - als Appell zum Gewissenerforschen des Abendlandes. Schönere Zukunft. Wochenschrift für Kultur und Politik, Volkswirtschaft und Soziale Frage V(36):857-858. 8.6.1930.

Schmidt, W. 1930e. Handbuch der vergleichenden Religionsgeschichte. Ursprung und Werden der Religion. Theorien und Tatsachen. Münster: Aschendorff.

Schmidt, W. 1937. Handbuch der Methode der kulturhistorischen Ethnologie. Münster: Aschendorff.

Schmidt, W. 1955. Der Ursprung der Gottesidee. Eine historisch-kritische und positive Studie. Münster: Aschendorff. 12 Bände, 1912-1955.

Schmidt, W., und W. Koppers (Hrsg.). 1924. Völker und Kulturen. Erster Teil: Gesellschaft und Wirtschaft der Völker. Regensburg: Habbel.

Stehle, H. 1993. Geheimdiplomatie im Vatikan. Die Päpste und Kommunismus. Zürich: Benzinger.

Turi, G. 2002. Il mecenate, il filosofo e il gesuita. L' „Enciclopedia italiana“, specchio della nazione. Bologna: Il Mulino.

Walk, L. 1928. Eine wissenschaftliche Abrechnung mit Freud und seiner Lehre. Die ethnologischen Grundlagen der Psychoanalyse. Ein Vortrag Professor P. W. Schmidts. Reichspost. Unabhängiges Tagblatt für das christliche Volk 35(335):14. 2.12.1928. 
Wassermann, J. 2014. Black Vienna. The radical right in the red city. Ithaca: Cornell University Press. Weiss, E. 1931. Elementi di Psicoanalisi, prefazione di Sigmund Freud. Milano: Hoepli.

Yerushalmi, Y.H. 1993. Freuds Moses - Endliches und unendliches Judentum. Berlin: Wagenbach. orig. 1991.

Zapperi, R. 2016. Freud und Mussolini. Berlin: Berenberg. Aus dem Italienischen von Ingeborg Walter. Zettler, D. 2020. Das Maßlose der Spätmoderne: Eine Kritische Theorie. Bielefeld: transcript. 